\title{
SPICES, COVID-19 AND LOW HANGING FRUITS FOR THE PHARMACEUTICAL INDUSTRY
}

Dear Reader,

Since the time the COVID-19 pandemic hit, the whole world is racing to develop vaccines. Vaccines that are in the development stage are also not a panacea. Preliminary studies have revealed that the 'antibodies' formed post vaccinations do not last long, vaccination with some of them resulting in potential protection for just 2 to 3 months. This would mean a need for repeated vaccinations? Concurrently, some scientists thought that vaccination of the Asian Population with BCG Vaccines, may have played a role to reduce the incidence and morbidity due to COVID-19 in Asian nations and the data for India on the spread, recovery and mortality reflect this to some extent.

In many Asian nations and in India, it is common advice by grandmothers to prescribe "Pepper Rasam", (a form of light soup with lentils and pepper) to ward off allergic rhinitis and viral attacks. I vouch for its effectiveness having experienced its benefits umpteen times to stop my running nose, viral attack, and low immune status. Regular consumption of spices in small quantities are known to benefit to keep healthy and fight various types of viral attacks.

What about Spices consumed in these nations? This question has been evaluated on the basis of scientific data. Elsayed and co-workers* exactly looked at the potential correlation between COVID-19 and spice consumption. COVID-19 data from 163 countries including total cases, total deaths, and total recovered were reviewed after due normalizations to population numbers and other factors. The consumption figures of spices in these nations were looked into. A clear interrelated prevalence between the total number of COVID-19 cases per million populations and the grams of spice supply per capita per day were seen. Nations with lower consumptions of spices per capita showed greater number of COVID-19 cases per million populations. Only exceptions were Luxemburg and Iceland which showed greater number of COVID-19 cases relative to the trend. Although it is widely accepted that there is no genetic pre-disposition and SARS-CoV-2, is capable of affecting all races, the mortality rate associated with COVID-19 varies among different ethnic groups. For example, COVID-19 fatalities in New York City were considerably less among South Asians compared to other ethnicities. A breakdown of COVID-19 fatalities among Whites, Hispanics, Blacks, and Asians was $27 \%, 34 \%, 28 \%$, and $7 \%$ compared to their populations of $32 \%, 29 \%, 22 \%$, and $14 \%$, respectively. Interestingly, Asians suffered from only $7 \%$ of the COVID-19 fatalities despite their $14 \%$ population in the city. In this regard, Asians had a 50\% lower than expected fatality rate. Elsayed reported that "the nations which had lower consumption of spices per capita, had higher number of deaths per million.

A number of spices have demonstrated their immunity enhancing benefits and published scientific literature is full of data. Capsicum (Chillies), ginger, turmeric, cinnamon, cumin, fenugreek and black pepper are known to work on T-Cells, CD counts, provide anti-inflammatory effects through action on Interleukins and other immune markers. Pepper and Cinnamon show their effect on respiratory conditions. Garlic another spice widely consumed is known for its anti- bacterial and anti-viral properties.

No wonder, India which is a source for a number of spices saw a growth in the export of spices in the last few months. Most Indians are also drinking one or the other recipe of spices 
as "Kadha" (an herbal decoction as tea) ever since COVID-19 hit and one wing of the Ministry of Health recommended to drink such a kadha with basil, cinnamon, ginger and pepper daily.

The pharmaceutical industry seems to be in their own world in pursuit of vaccines or repurposing drugs approved in other nations for generic use. Use of Social distancing, wearing masks and washing of hands have all played their role in reducing the impact of COVID-19 to whatever extent they have. Prevention is playing the role primarily advocated by all experts. Products that can enhance innate immunity using spices and their extracts can be a good methodology to support the preventive approach.

Entry of the virus to the body via air borne droplets is through the oral route and nasal route. Development of an effective oral gargle or a mouth wash and an effective nasal spray or drops that can give a lining to the nostrils and "catch \& kill" the virus within the oral cavity and nose respectively are additions to these measures. An oral gargle liquid formulated with distilled oils or extracts of selected spices that have antibacterial activity can be thought of and achieved. Formulated with such natural actives, they need not be "spit out" but swallowed post washing or gargling. The stomach's acidic nature is adequate to further kill and destroy the bacteria and the viruses that might have entered the mouth. Number of actives are available for such a use and selection carefully and good formulation methodology will potentially also be IPR protectable. Even a well formulated "spray like a mouth freshener" can be another option for such a product. Formulation of a nasal spray or a drop with 'oleaginous' base can be ideal for giving a good coating to the nostril's lining. Consideration of distilled oils, that dissolve in vegetable oils offer a wide range of actives from the natural kitty. If one does not want to consider natural actives, anti-microbial actives from the synthetic molecule libraries can be used. In a pandemic situation, consumers would be willing to do many things and asking them to use a mouth wash and apply a nasal drop would be convincing given the marketing muscle of the pharmaceutical and FMCG firms. These are low hanging fruits that seems to have missed the Research \& Development heads and the business heads, perhaps in their quest for "Curative and big ticket items".

Would some Indian Pharma firm, known for their Jugaadu ability and increasingly innovative strategy catch these fruits?

( ${ }^{*}$ ehaya Elsayed and Naveed Ahmed Khan, ACS Publications, https://dx.doi.org/10.1021/ acschemneuro.0c00239)

https://doi.org/10.53879/id.57.07.p0005

$\operatorname{Dr} D$ B Anantha Narayana Bengaluru

\section{About The Guest Editor}

$\operatorname{Dr} D$ B Anantha Narayana, is the Chief Scientific Officer, AYURVIDYE TRUST, Bangalore. He Championed the Notifications of Supplements and Nutraceuticals Regulations, FSSAI, 2016 Updated in 2017 and Phytopharmaceuticals as Drugs under Drugs \& Cosmetics Act \& rules, 2016. He is a recipient of Indian Drugs award for Contribution to IDMA and Indian Drugs and is a recipient of Eminent Pharmacist's Award of IPA, 2007. Currently is an expert member, amongst others contributing significantly to 1) MemberExpert committee - Non-Specified Foods \& Food Ingredients - FSSAI, 2) Chairman-Expert Committee -Advertisement and Claims - FSSAI, 3) Chairman-Scientific Panel - Nutraceuticals of FSSAI. 4) Chairman - Phytopharmaceuticals and Herbal products of Indian Pharmacopeia Commission 5) Member-Steering Committee of NMPB, Ministry of Ayush.

He continues to guide youngsters in research and also guides many startup firms in the area of Supplements/ Nutraceuticals, Foods, herbals and cosmetics.

प

If you would like to comment on the editorial please write to us at publications@idmaindia.com 